\title{
Fire Tracking in Video Sequences Using Geometric Active Contours Controlled by Artificial Neural Network
}

\author{
Aymen Mouelhi \\ LR13ESO3, SIME \\ University of Tunis, ENSIT \\ Tunis, Tunisia \\ aymen_mouelhi@yahoo.fr \\ Eric Moreau \\ CNRS, LIS \\ Aix Marseille Univ., Université de \\ Toulon \\ Toulon, France \\ moreau@univ-tln.fr
}

\author{
Moez Bouchouicha \\ CNRS, LIS \\ Aix Marseille Univ., Université de \\ Toulon \\ Toulon, France \\ moez@univ-tln.fr
}

\author{
Mounir Sayadi \\ LR13ES03, SIME \\ University of Tunis, ENSIT \\ Tunis, Tunisia \\ mounir.sayadi@esstt.rnu.tn
}

\begin{abstract}
Automatic fire and smoke detection is an important task to discover forest wildfires earlier. Tracking of smoke and fire in video sequences can provide helpful regional measures to evaluate precisely damages caused by fires. In security and surveillance applications, real-time video segmentation of both fire and smoke regions represents a crucial operation to avoid disaster. In this work, we propose a robust tracking method for fire regions using an artificial neural network (ANN) based approach combined with a hybrid geometric active contour model (GAC) based on Bayes error energy functional for forest wildfire videos. Firstly, an estimation function is built with local and global information collected from three color spaces (RGB, HIS and YCbCr) using Fisher's Linear Discriminant analysis (FLDA) and a trained ANN in order to get a preliminary fire pixel classification in each frame. This function is used to compute initial curves and the level set evolution parameters to control the active contour model providing a refined fire segmentation in each processed frame. The experimental results of the proposed tracking scheme proves its precision and robustness when tested on different varieties of scenarios whether wildfire-smoke video or outdoor fire sequences.
\end{abstract}

Keywords- Fire detection; color spaces; Fisher's linear discriminant analysis; neural networks; geometric active contours.

\section{INTRODUCTION}

Wildfire is an uncontrolled fire in a region of flammable flora that occurs in the countryside area. It differs from other fires by its wide dimension, the speed at which it can spread out from its original source, its potential to alter direction unpredictably, and its ability to skip gaps such as rivers, roads and fire breaks. Forest fire is distinguished in terms of the cause of detonation, by their physical characteristics such as velocity of propagation, the existing combustible material, and the weather effect on the fire [1]. In the last few years $[2,3]$, research was focused on modeling and segmenting fire regions using several image processing techniques but nowadays, it has been shifted to track its motion to predict the fire evolution and the eventual directions that can be taken in video sequences [4-8].

Different techniques of real-time fire detection are presented in the literature with the help of computer vision algorithms and can be used by capturing the images from surveillance systems or drones with camera monitoring indoors and outdoors [1]. Generally, they can be separated into either flame detection or smoke detection in terms of the real object being extracted [8]. In the case of detect fire in large and open spaces, a lot of study have shown that video based fire detection methods are well appropriate to analyze the temporal activities and perform a three dimensional localization of the fire [4-8]. In this context, it would be very interesting to implement a real-time video fire tracking in order to study flame's behavior in time and space domain.

Generally, the color, motion, and geometry of the fire represent the three main features of fire detection [2]. In the literature, the common proposed video-based fire detection approaches relies on the color and the morphology characteristics joined with the temporal behavior of flames [3-6] [8,9]. In fact, the main objective is to design a rulebased scheme or a multi-dimensional feature vector which is used to feed a conventional classification method such as: artificial neural networks (ANN), support vector machine (SVM), Bayesian learning, etc. Indeed, the performance of these approaches is hardly relied on the expert knowledge to extract the significant features and to choose the best technique to be used for the learning process. On the other side, some other approaches appears recently and they don't need manual intervention of experts to extract the relevant features of the detected objects in the videos. Most of them are based on deep neural networks learning [7] [10,11] and they need a big image dataset for training to attempt good results in the real-time detection process.

Thus, in this work we present a new approach that combines pixel classification using ANN and geometric active contour model in order to track fire evolution in video sequences. A multilayer neural network is constructed here and trained by transformed color and textural features using the advantages of Fisher's Linear Discriminant analysis. Then, a geometric active contour model is implemented and controlled by the network classification results to provide fast fire region's segmentation in each frame in the processed video.

The remainder of the paper is organized as follows: In section II, we present a brief review of related works. The 
next section describes the proposed fire tracking method. Section IV reports the experimental results and performance analysis. Finally, summary and conclusions are provided in section $\mathrm{V}$.

\section{RELATED WORK}

Several works have provided a significant contribution in developing constructive real-time video fire detection algorithms. In the work of Celik et al. [2], a fire detection algorithm is proposed that combines color information of fire with temporal changes, and background subtraction assisted foreground object segmentation. Poobalan et al. [3] proposed a fire detection scheme based on image processing techniques which can be applied in numerous surveillance devices. The algorithm combines color and edge information using RGB and HIS spaces to segment fire. Other promising detection approaches $[9,10]$ are presented to fire region identification in video sequences using multidimensional local and/or features combined a supervised classifier. In [10] for example, authors used local, geometric and statistical features such as color, area size, boundary roughness and skewness of the potential fire regions to describe flames. Then, discriminant features are provided to Bayes classifier as an input vector to achieve a decision about the fire existence. However, as we said previously, these methods needs a prior knowledge about features that characterize fire from the other objects and needs to use preprocessing techniques to extract discriminative ones. Recently, deep learning algorithms is gaining more and more interest since they don't require the attribute extraction phase. Frezzi et al. [7] developed a deep convolutional neural network $(\mathrm{CNN})$ for fire and smoke detection. Fire/smoke recognition is obtained overall each frame by applying a sliding window on the feature map generated with the network. In the work of Khan et al. [11], a CNN architecture is inspired by SqueezeNet and designed for fire detection and localization in fire scenarios. The network uses smaller convolutional masks and lightened fully connected layer in order to increase the computational performance. Kim et al. [12], proposed a new method that uses Faster Region-based CNN to identify the suspected regions of fire and of non-fire based on their spatial features. A further solution is described in this work by combining the new discriminative features for fire detection using FLDA-ANN classifier and the advantages of the GAC method for fire localization, and semantic understanding in video sequences.

\section{The Proposed Fire Tracking MethoD}

The goal of the proposed tracking method is the boundary contour detection of the fire regions in each frame of the video. This task is completed by the use of a hybrid geometric active contour model combined with a fire feature classification method based on ANN and Fisher's linear discriminant preprocessing. As illustrated in Fig. 1, fire detection regions in video sequences is composed of the following stages:

Learning: In the first stage, a learning dataset is built using image patches sized $100 \times 100$ pixels from two main classes: Fire and background. In fact, each pixel is represented in three color spaces (RGB, HIS and $\mathrm{YCbCr}$ ) in order to compute intensity and textural features that characterize flames. Then, the original variables of the training set are analyzed and reduced to uncorrelated components using FLDA strategy similar to that described in
[13]. The resulting Fisher features $\left(F_{1}, F_{2}, \ldots, F_{c-1}\right)$ are presented then to feed-forward neural network with their desired outputs for learning.

Processing: The second stage consists mainly of three parts: Video decomposition to RGB frames, feature extraction and classification and fire detection by geometric active contours.

\section{A) Feature extraction and classification using FLDA and ANN}

To obtain initial segmentation of fire areas in each frame, a feature classification approach is performed using a learning technique based on FLDA and ANN classifier. In the processing stage, pixels of the processed frame are organized according to their most relevant features obtained from RGB, HIS and YCbCr color spaces. The transformed feature values obtained with the FLD analysis are provided to the trained network in order to get the discrimination function $\left(\alpha_{F}\right)$ that estimate the location of the flames in the current frame (see Fig. 1).

1) Fisher's Linear Discriminant analysis (FLDA) strategy: For fire image recognition, we have selected simply 8 features based on chromatic and textural information based on three color spaces that proved a good characterization in several works [2-4, 9]. It's known that flames reveal reddish colors which can be seen obviously in the Red component compared to the other channel of the RGB space. In fact, in fire regions, the intensity value of the Red channel is greater than the Green and the Blue value intensities [9]. For this reason, the first feature that we will be used to recognize fire in image patches is the Red component. Then, to provide more information about the pixel intensity and its neighborhood in a fire blob, we have added 2 statistical measures obtained from the Red channel which are mean Red value and the variance Red value of a pixel neighborhood. Indeed, these global features will takes into consideration the illumination changes and they can be very relevant for pixel classification. In our work, a sliding window centered around every pixel and sized $9 \times 9$ is used to extract local mean and variance images of Red Channel (see Fig. 2(b)). This provides more vigorous representation for fire pixels. So, it is needed to transform RGB color space to other spaces where the discrimination between intensity and chrominance is more appropriate $[4,9]$. By converting RGB to $\mathrm{YCbCr}$ space, it can be clearly observed an important difference between the chrominance blue $\mathrm{Cb}$ and the chrominance red $\mathrm{Cr}$ from a representative fire image. The same thing can be noticed when evaluating the difference between the luminance $\mathrm{Y}$ and the chrominance $\mathrm{Cr}$ (Figs. 2(c) and (d)). Consequently, two features can be extracted from the $\mathrm{YCbCr}$ color space and they are formulated as follow:

$$
\begin{aligned}
& |Y(x, y)-C b(x, y)| \\
& |C b(x, y)-C r(x, y)|
\end{aligned}
$$

In addition, HIS color model is frequently adapted due to its convenience for providing a more people-oriented manner of describing the colors [4]. In addition to the RGB and $\mathrm{YCbCr}$ features mentioned above, we have used also the 
three components of HIS color space as color attributes in the proposed pixel classification approach.

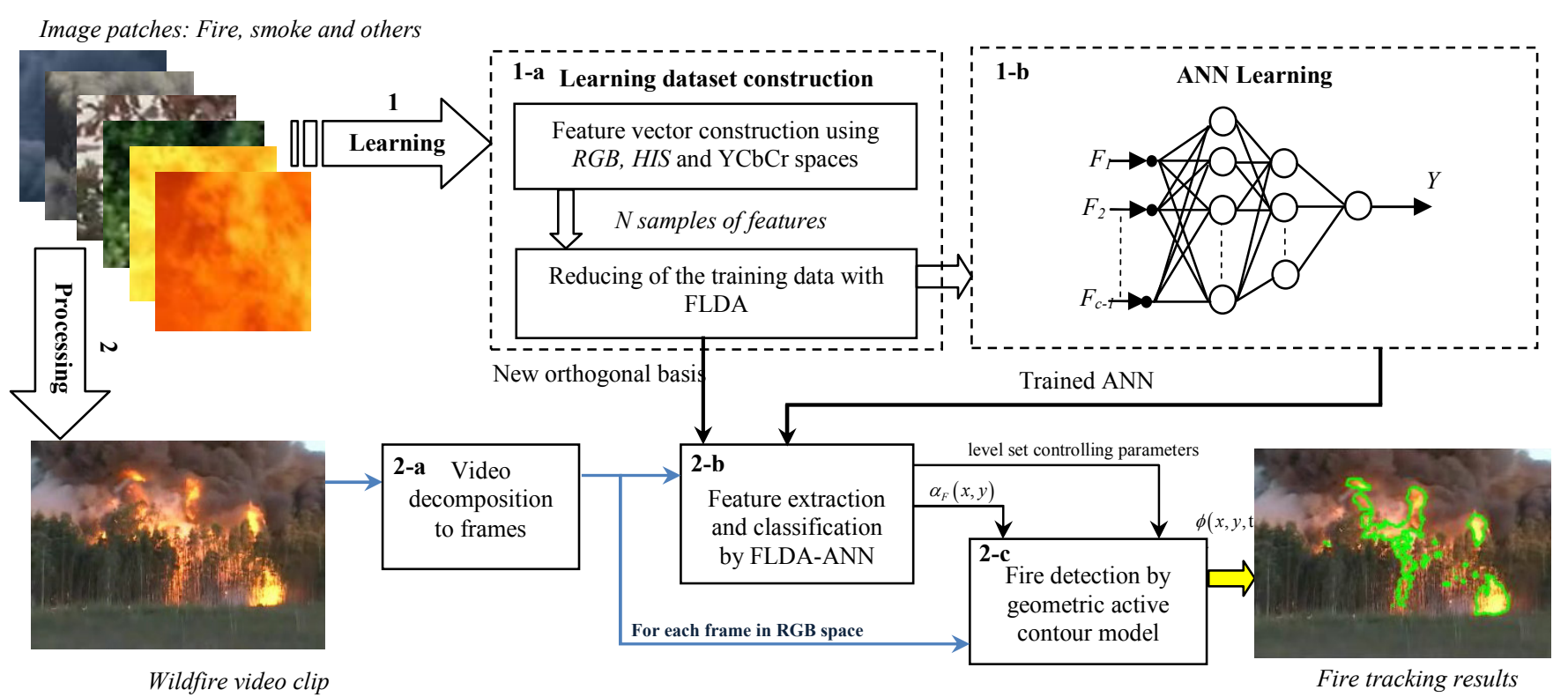

Fig. 1: Illustration of the proposed fire tracking process.

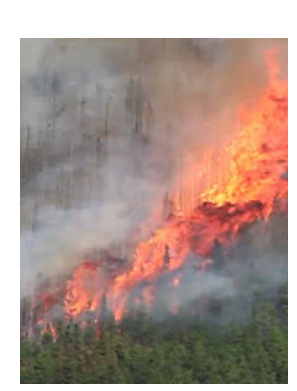

(a)

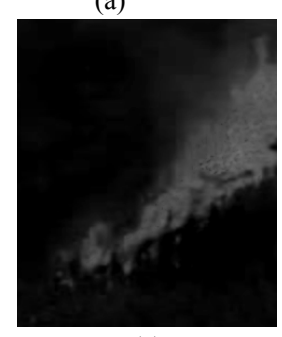

(c)

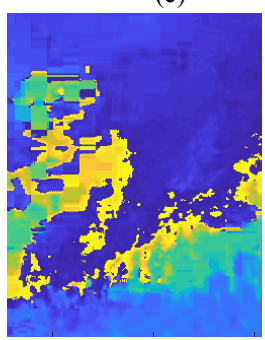

(e)
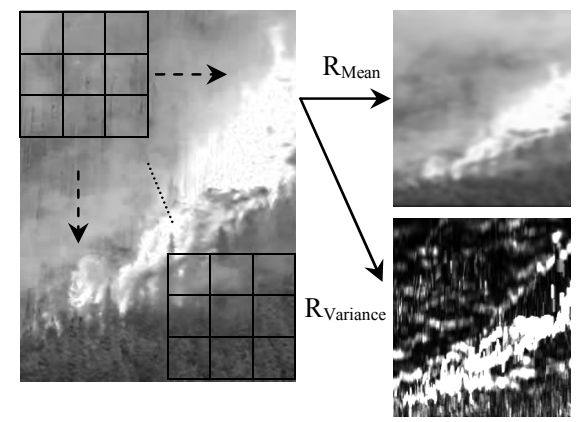

(b)

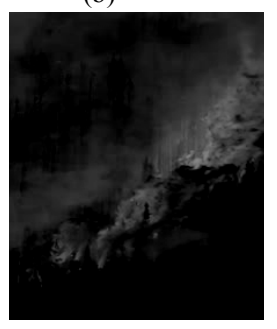

(d)

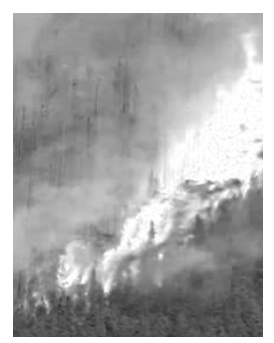

(f)

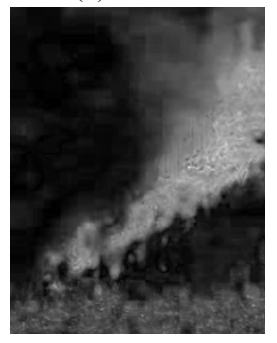

(g)
Fig. 2: Chromatic and textural feature extracted for classification: (a) $R G B$ original image, (b) Mean and variance extraction of Red Channel, (c), (d) absolute difference between channels of $\mathrm{YCbCr}$ space as defined in Eq. (2) and (1) respectively. (e), Hue, (f) Intensity and (g) Saturation component of HIS color space.

To train the neural network with the extracted features, a new preprocessing approach inspired by the idea of X. Long et al. [13] is adopted in this work using FLD. In fact, the FLDA is used to generate new independent and significant features by reducing the dimension of the initial feature space to no more than $\mathrm{c}-1$, where $\mathrm{c}$ is the number of classes [13-15]. In our work, pixels in the current frame can belong to one of two classes : "Fire" and "Non-Fire". Thus, if FLDA is directly applied, the resulting subspace can only be onedimensional, which is not enough for a compound segmentation problem like fire detection. Therefore, we propose to treat fire pixel classification as a multiclass problem in the preprocessing step by dividing the "Non-Fire" class to multiple subclasses as illustrated in Fig. 3. This is possible since the "Non-Fire" class includes a various set of image patches of non-fire objects such as 'smoke', 'sky', 'buildings', 'trees', etc. Thereby, the size of the subspace created by FLDA is increased to be equal to ("Non-Fire" classes + "Fire" class - 1). The input learning data is divided into three subsets corresponding to the object class c: one "Fire" class and 6 "Non-Fire" classes. The use of FLDA in this case would be very beneficial because it reduces variation within individual "Non-Fire" subclasses.

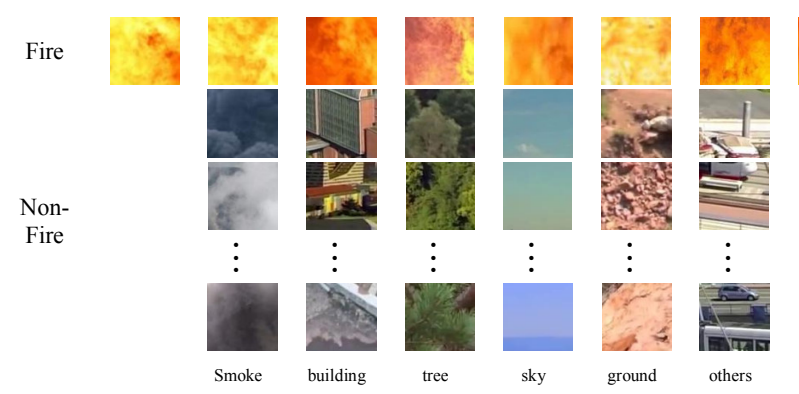

Fig. 3: Illustration of image patches sized $100 \times 100$ in the learning stage using the new strategy of FLD preprocessing for feature classification.

2) Feature classification: To classify extracted features represented in the new subspace given by the FLD preprocessing, we trained a feed-forward ANN using the quasi-Newton algorithm. In this framework, the ANN structure contains six inputs, three hidden layers and a single neuron in the output layer. The transfer function throughout the network is the hyperbolical tangent sigmoid function. 
The output node is intended to label each pixel to one of two classes: "Fire" pixel or "Non-Fire" pixel. So, a target array is created by $\mathrm{N}$ elements, where $\mathrm{N}$ is the number of feature samples . The ANN output values are either 1 ("Fire" class) or -1 (Non-Fire class). The tangent sigmoid activation function is used for all neurons. The training patterns are constructed by computing color and statistical features for each image sample and converted subsequently to 1Dvectors. These input vectors are organized according to their output label in a learning matrix $\mathrm{Xin}=(\mathrm{F} 1, \mathrm{~F} 2, \ldots, \mathrm{F} 6)$. The number hidden is chosen in each layer empirically during the learning process. More information about this and other training/ validation details are described in the next section.

In the processing stage, after decomposing the fire video to RGB frames, all frames are processed iteratively by the proposed approach based on fire feature classification and geometric active contour model. First, all extracted pixel features of each frame are transformed to FLD components. Then, they are provided to the trained neural network for classification. At the end, a binary image $\alpha_{F}$ that estimates fire regions is obtained by thresholding of the output value for each pixel in the frame:

$$
\alpha_{F}(x, y)= \begin{cases}1 & \text { if } Y(x, y) \geq s_{0} \\ 0, & \text { otherwise }\end{cases}
$$

where $s_{0}$ is an variable threshold which is fixed to estimate the interesting regions. $\mathrm{s} 0$ is set to 0 in this work. Example of fire segmentation by the FLDA-ANN classifier is displayed in Fig. 4(b). The final step of the proposed fire tracking scheme is the contour detection of fire regions using a modified active contour model similar to the model presented in [15]. This task is applied for each frame of the video clip to extract boundaries and areas of fire in the sequence.

\section{B) Fire tracking by geometric active contours}

To recover the classification results using FLDA-ANN method, we propose in this paper to detect fire boundaries by geometric active contour model (GAC) inspired from the work of A. Mouelhi et al. [15]. This model is employed to segment automatically fire contours starting from the pixel classification results obtained by the neural network classifier in each frame of the video. Moreover, the parameters that control the curve evolution are computed directly from the classification results.

The GAC proposed here is a combination of the classical geodesic active contour model and two-region segmentation model based on Bayes error energy functional [16].

To extract object boundaries in a color image with the classical geodesic active contour, a curve $C$ should be create which minimizes the energy functional below:

$$
E_{C}(C)=\int_{0}^{L(C)} g_{c o l}(C(q)) d s+v \int_{\Omega} g_{c o l}(C(q)) d A
$$

where $\Omega \subset \mathfrak{R}^{2}$ is the 2-D domain of the color image. $C(q)=(x(q), y(q))$ is a differentiable closed curve in $\Omega$ with a position parameter $q \in[0,1]$ and Euclidean length $L$. The distance $d s$ is the Euclidean metric, $d A$ is the element of area and $v$ is a fixed parameter that controls the area of the curve. $g_{c o l}$ is an edge-detector for color images [15]. The general curve evolution along its normal direction $\vec{N}$ with speed $F$, is $\frac{\partial C}{\partial t}=F \cdot \overrightarrow{\mathrm{N}}$. The numerical solution of the curve evolution problem is obtained by the fast level set formulation proposed in [16]:

$$
\left\{\begin{array}{l}
\frac{\partial \phi}{\partial t}=\delta_{\varepsilon}(\phi)\left(\lambda d i v\left(g_{c o l} \frac{\nabla \phi}{|\nabla \phi|}\right)+v g_{c o l}\right)+\mu \operatorname{div}\left(\left(1-\frac{1}{|\nabla \phi|}\right) \nabla \phi\right) \\
\phi(x, y, 0)=\phi^{0}(x, y)
\end{array}\right.
$$

where $\phi$ is a level set function defined on $\Omega$, whose zero-level set $C=\{(x, y) \mid \phi(x, y)=0\}$ defines the boundary contour of the objects such that $\phi>0$ inside the segmented objects and $\phi<0$ outside. $\mu$ is a positive parameter that control the penalization term $\operatorname{div}((1-1 /|\nabla \phi|) \nabla \phi)$ which is used to maintain the signed distance property $|\nabla \phi|=1$. $\delta_{\varepsilon}(\phi)$ denotes the regularized Dirac function with width controlled by $\varepsilon$ [17]. $\lambda$ is a constant controlling the curvature term and $\phi^{0}(x, y)$ is the initial contour. Then, Bayes error based two-region segmentation energy functional is added to the classical model in order to enhance segmentation precision by integrating more global information inside and outside the contour in the level set formulation [15]. The region energy functional is formulated as follow:

$$
E_{R}(\phi)=-\int_{\Omega}\left(H_{\varepsilon}(\phi) \log \left(P_{1}\right)-\left(1-H_{\varepsilon}(\phi)\right) \log \left(P_{2}\right)\right) d x d y
$$

where $P_{l}=P\left(z \mid \Omega_{1}\right), \quad P_{2}=P\left(z \mid \Omega_{2}\right) \quad$ are the probability densities of the regions $\Omega_{1}$ (inside $C$ ) and $\Omega_{2}$ (outside $C$ ) and $H_{\varepsilon}(\phi)$ is the regularized Heaviside function. We used Gaussian density functions for $P_{1}$ and $P_{2}$. The energy $E_{R}(\phi)$ is minimized with respect to $\phi$ according to the gradient descent rule and it is given by:

$$
\frac{\partial \phi}{\partial t}=\delta_{\varepsilon}(\phi) \log \left(\frac{P_{1}}{P_{2}}\right)
$$

Combining both level set formulations (Eqs. (5) and (7)), we get the improved geometric active contour formulation for color image expressed by:

$$
\begin{aligned}
\frac{\partial \phi}{\partial t}= & |\nabla \phi|\left(\gamma \log \left(\frac{P_{1}}{P_{2}}\right)+\lambda \operatorname{div}\left(g_{c o l} \frac{\nabla \phi}{|\nabla \phi|}\right)+v g_{c o l}\right) \\
& +\mu \operatorname{div}\left(\left(1-\frac{1}{|\nabla \phi|}\right) \nabla \phi\right)
\end{aligned}
$$

here $\delta_{\varepsilon}(\phi)$ is replaced by $|\nabla \phi|$ in order to intensify the effective range in the whole image [16]. $\gamma$ is a positive parameter which handles the region term. Finally, the discrete scheme of the level set function is given by:

$$
\phi^{k+1}(x, y)=\phi^{k}(x, y)+\tau L\left(\phi^{k}(x, y)\right)
$$

where $L\left(\phi^{k}(x, y)\right)$ is the numerical estimation of the righthand side in (8) and $\tau$ is the time step.

In addition, the initial curve and the stopping function in each frame are automatically computed using the 
preliminary classification results given by $\alpha_{F}$. The initial fire curves $\phi_{F}^{0}$ are expressed by:

$$
\phi_{F}^{0}=4 \varepsilon\left[\left(G_{\sigma} * \alpha_{F}\right)-0.5\right] \text {, }
$$

where $G_{\sigma} * \alpha_{F}$ is the Gaussian convolution of the discrimination function that estimates the location of flames.

The evolution parameters of the level set function are determined adaptively from the initial fire curves $\phi_{F}^{0}$. Generally, the level set evolution will be faster if the object of interest is big. So, the time step $\tau$ is assigned to the ratio $a / \ell$, since it will be big for large objects. Time step corresponding to the level set function is obtained by:

$$
\tau=\frac{a_{F}}{\ell_{F}}=\frac{\int_{I} H_{\varepsilon}\left(\phi_{F}^{0}\right) d x d y}{\int_{I} \delta_{\varepsilon}\left(\phi_{F}^{0}\right) d x d y}
$$

where $a_{F}$ and $\ell_{F}$ represent respectively the area and the length of the estimated fire regions. $\mu, \lambda$ and $\gamma$ are also resultant from the preliminary classification results and calculated by :

$$
\mu=0.2\left(a_{F} / \ell_{F}\right)^{-1}
$$

and

$$
\gamma=\lambda=0.1\left(a_{F} / \ell_{F}\right)
$$

To compute the balloon force $v$ which is used to determine the advancing direction of the level set function, we have used the ANN classification output for each pixel in the processed frame:

$$
v(x, y)=Y(x, y)
$$

The new balloon force such that $-1 \leq v(x, y) \leq 1$ is a matrix in which the sign of value at each pixel identifies the manner of the level set evolution: positive for shrinkage and negative for expansion. Fig. 4(c) and 4(d) show initial and final fire contours obtained with the proposed GAC.

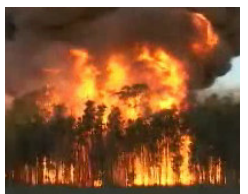

(a)

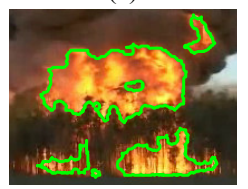

(c)

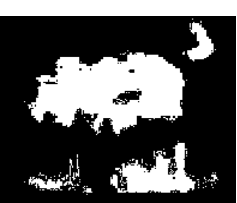

(b)

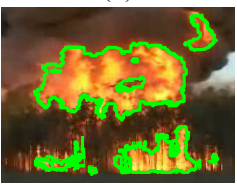

(d)
Fig. 4: Main steps of boundary fire detection for a processed frame: (a) RGB frame, (b) initial pixel classification using FLDA-ANN classifier, (c) and (d) fire contour detection results using GAC after 2 and 10 iterations, respectively.

\section{EXPERIMENTAL RESULTS}

The fire video data studied in this work contains 31 sequences including 62690 RGB frames. The video clip database two type of videos captured in different environment, where 14 videos include a fire, while 17 are normal videos (see [11] for more details). In our experiments, we choose to test some of the normal videos including fire-like objects which makes fire detection more challenging, and hence classical fire recognition methods may wrongly classify these frames. The performance of the proposed tracking scheme is performed by evaluating the obtained results in terms of pixel classification using FLDAANN method and boundary fire detection by the proposed GAC model.

The best training and validation performance of the FLDA-ANN classifier is obtained after several experiences. In fact, to train the network we have used 100 RGB image patches $(100 \times 100$ pixels $)$, where 30 images correspond to the "Fire" class and 70 images represent the "Non-Fire" class. $15 \%$ of the overall image patches are extracted and used for validation and $10 \%$ for test data. The structure of the ANN (number of hidden nodes) is optimized empirically by independently varying the number of hidden neurons in each layer, and evaluating the generalization properties of the network on the validation set at each step. So, we found that, 32 neurons in the first layer, 16 and 8 neurons in the second and the third layer respectively, provide the best performance in term of the mean squared error for training, validation and testing phases (see Fig. 5).

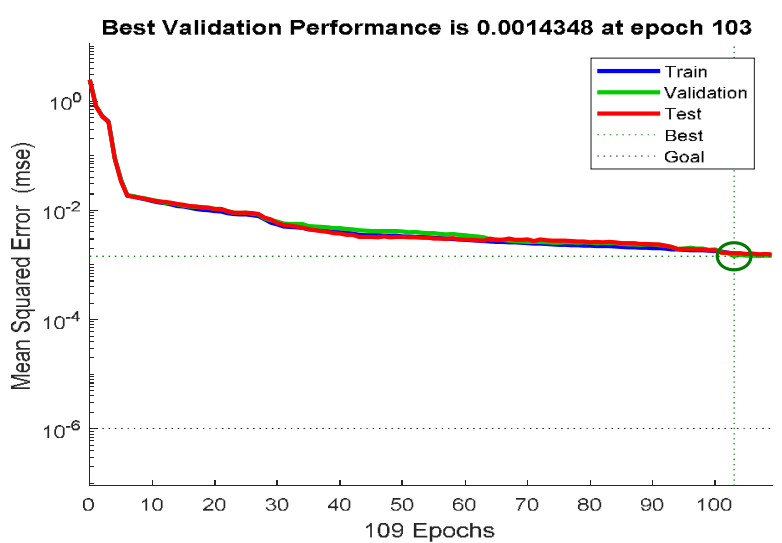

Fig. 5: Training, validation and test performance evolution of the FLDAANN classifier.

In the other hand, performance of the proposed fire tracking scheme is tested on the whole video clip database and compared with the Celik et al. [2] and Khan et al. [11]. The fire detection accuracy is evaluated in this work by computing false positives and false negatives and detection accuracy rate. Table I shows that the proposed method is more efficient than the classical algorithm of Celik et al. [2] while the CNN approach of Khan et al. [11] still dominant in terms of False positives, negatives and detection rate. Nevertheless, our approach is very closer in terms of detection results and could be improved by integrating additional training scenarios.

In order to illustrate qualitatively the performance of our fire tracking method on the fire/non-fire video sequences, we choose 5 frames starting at frame 40 to 240 with sampling step of 50. The final fire contours using the proposed scheme are represented in Fig. 6. Furthermore, semantic segmentation of a set of frames extracted from the fire video data is evaluated using Jaccard index [15]. In fact, we selected 5 frames from each fire sequence and compared with manually segmented frames. Table II report fire segmentation evaluation in term of Jaccard similarity measure on 70 frames of our database. 


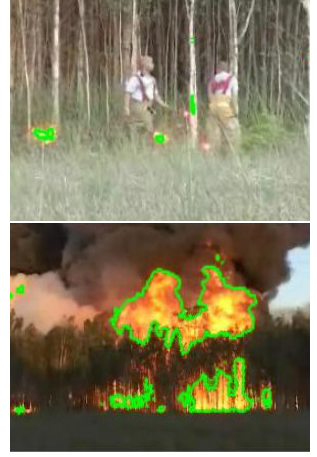

(a) frame 40

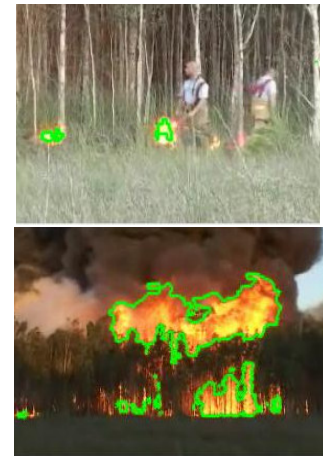

(b) frame 90

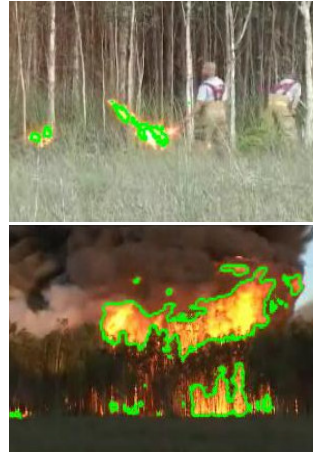

(c) frame 140

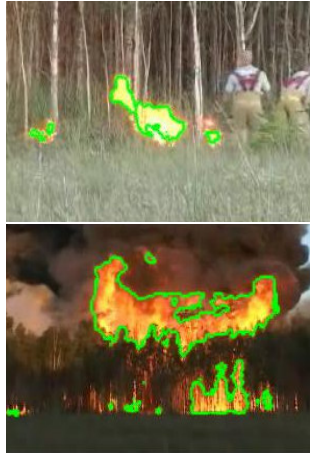

(d) frame 190

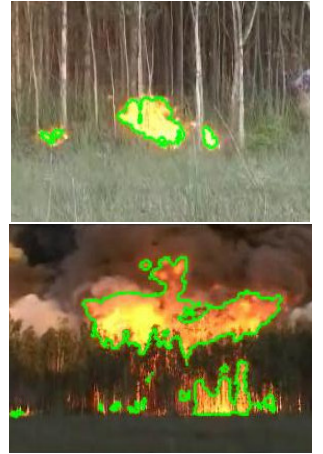

(e) frame 240

Fig. 6: Fire tracking results of the proposed method of subsequent frames extracted from two different videos illustrated in raw(1) and raw (2) : final fire boundary detection results in (a) frame 40, (b) frame 90, (c) frame 140, (d) frame 190 and (e) frame 240.

TABLE I. COMPARATIVE FIRE DETECTION RESUlts ON THE VIDEO SEQUENCES DATA

\begin{tabular}{|c|c|c|c|}
\hline & $\begin{array}{c}\text { Celik et al. } \\
{[\mathbf{2}]}\end{array}$ & $\begin{array}{c}\text { Khan et al. } \\
{[\mathbf{1 1}]}\end{array}$ & $\begin{array}{c}\text { Proposed } \\
\text { method }\end{array}$ \\
\hline False positives (\%) & 29.41 & 8.87 & 10.1 \\
\hline False negatives (\%) & 0.00 & 2.12 & 5.3 \\
\hline Accuracy (\%) & 83.87 & 94.50 & 93.2 \\
\hline
\end{tabular}

TABLE II. EVALUATION OF FIRE SEGMENTATION RESUlts USING JACCARD MEASURE

\begin{tabular}{|c|c|c|c|c|}
\hline & Mean & Variance & Min & Max \\
\hline Proposed method & 90.1 & 3.1 & 80.9 & 97.5 \\
\hline
\end{tabular}

\section{CONCLUSION}

In this paper, we have proposed a fire tracking method based on artificial neural network and geometric active contour model. To get initial pixel classification of flames in each frame, a discrimination function is constructed using a ANN combined with a new FLD preprocessing. This function is used to control the GAC evolution in order to obtain fire boundaries in reasonable processing time. The proposed method provides encouraging segmentation results achieving $90.1 \%$ using of Jaccard similarity measure. In view of the GAC method's reasonable accuracy for fire detection $(93.2 \%)$ and the rate of false alarms, the tracking system can be helpful to disaster supervision teams in controlling fire disasters in a timely way, thus avoiding enormous fatalities.

As future work, we intend to improve the method by the use of CNN to extract features automatically and to compute the controlling parameters of a faster GAC model. In addition, the proposed scheme can be extended to detect and localize smoke in a wider variety of video fire images for early fire recognition.

\section{REFERENCES}

[1] C. Yuan, Y. Zhang, Z. Liu, "A Survey on Technologies for Automatic Forest Fire Monitoring, Detection and Fighting Using UAVs and Remote Sensing Technique", Canadian Journal of Forest Research, March 2015, DOI: 10.1139/cjfr-2014-0347.

[2] T. Celik, H. Demirel, H. Ozkaramanli, M. Uyguroglu, "Fire detection using statistical color model in video sequences", J. Vis. Commun. Image R. Vol. 18, 176 - 185, 2007.

[3] K. Poobalan and S-C Liew, Fire Detection Algorithm using Image Processing Techniques, Proceeding of the 3rd International
Conference on Artificial Intelligence and Computer Science (AICS2015), October 2015, Penang, MALAYSIA, pp.12 - 13.

[4] C. Yu, Z. Mei, X. Zhang, "A real-time video fire flame and smoke detection algorithm", Procedia Engineering, Vol. 62, 891 - 898, 2013.

[5] O. Gunay, K. Tasdemir, B.U. Toreyin and A.E. Cetin, "Fire Detection in Video Using LMS Based Active Learning", Fire Technology, Vol. 46, 551-577, 2010.

[6] X-L. Zhou, F-X. Yu, Y-C Wen, Z-M. Lu, G.H. Song, "Early Fire Detection Based on Flame Contours in Video", Information Technology Journal, Vol. 9, N5, 899-908, 2010.

[7] S. Frizzi, R. Kaabi, M. Bouchouicha, J-M Ginoux, E. Moreau, F. Fnaiech, "Convolutional neural network for video fire and smoke detection", $42^{\text {nd }}$ Annual Conference of the IEEE Industrial Electronics Society, IECON 2016, pp. 877-882.

[8] M. Li, W. Xu, K. Xu, J. Fan, D. Hou, Review of Fire Detection Technologies based on Video Image, Journal of Theoretical and Applied Information Technology, 2013. Vol. 49 No.2 pp. 700-707.

[9] P.B Pagar, A.N. Shaikh, "Real time based Fire and Smoke detection without sensor by image processing", International Journal of Advanced Electrical and Electronics Engineering, 2013. Vol. 2 No.6 pp. 25-34.

[10] K. Borges, P. Vinicius, J. Mayer and E. Izquierdo, "Efficient visual fire detection applied for video retrieval", IEEE 16th European Signal Processing Conference, 2008.

[11] M. Khan, J. Ahmad, Z. Lv, P. Bellavista, P. Yang, S.W. Baik, "Efficient Deep CNN-Based Fire Detection and Localization in Video Surveillance Applications", IEEE Trans. Syst. Man Cybern. Syst., 2018, 99, pp. 1-16.

[12] B. Kim and J. Lee, "A Video-Based Fire Detection Using Deep Learning Models ", Applied Science, 2019, 9, 2862, pp. 1-19.

[13] X. Long, W. L. Cleveland, and Y. L. Yao, "A New Preprocessing Approach for Cell Recognition", IEEE Transactions on Information Technology in Biomedcine, Vol. 9, No. 3, 2005, pp. 407-412.

[14] A.M. Martinez, A.C. Kak, "PCA versus LDA", IEEE Transactions on Pattern Analysis and Machine Intelligence, Vol. 23, No. 2, 2001, pp. 228-233.

[15] A. Mouelhi, M. Sayadi, F. Fnaiech, K. Mrad and K.B. Romdhane, "Automatic Image Segmentation of Nuclear Stained Breast Tissue Sections Using Color Active Contour Model and an Improved Watershed Method", Biomedical Signal Processing and Control, Vol. 8, №5, pp. 421-436, 2013.

[16] Z. Ying, L. Guangyao, S. Xiehua, Z. Xinmin, "A geometric active contour model without re-initialization for color images", Image and Vision Computing, Vol. 27, 2009, pp. 1411-1417.

[17] C.M. Li, C.Y. Xu, C.F. Gui, M.D. Fox, "Level set evolution without re-initialization: a new variational formulation", in: IEEE Conference on Computer Vision and Pattern Recognition, San Diego, 2005, pp. 430-436. 